\title{
Reducing sedentary behaviour to decrease chronic low back pain: the stand back randomised trial
}

\author{
Bethany Barone Gibbs, ${ }^{1}$ Andrea L Hergenroeder, ${ }^{2}$ Sophy J Perdomo, ${ }^{1}$ \\ Robert J Kowalsky, ${ }^{3}$ Anthony Delitto, ${ }^{2}$ John M Jakicic ${ }^{1}$
}

- Additional material is published online only. To view please visit the journal online (http://dx.doi.org/10.1136/ oemed-2017-104732).

${ }^{1}$ Department of Health and Physical Activity, School of Education, University of Pittsburgh, Pittsburgh, Pennsylvania, USA

${ }^{2}$ Department of Physical Therapy, School of Health and Rehabilitation Sciences, University of Pittsburgh, Pittsburgh, Pennsylvania, USA ${ }^{3}$ Department of Health and Kinesiology, Texas A\&M University Kingsville, Kingsville, Texas, USA Dr Bethany Barone Gibbs, Physical Activity and Weight Management Research Center, University of Pittsburgh, Pittsburgh, PA 15216, USA; bbarone@pitt.edu

Received 25 August 2017 Revised 13 December 2017 Accepted 22 December 2017 Published Online First 12 January 2018

\section{Correspondence to}

\section{ABSTRACT \\ Objective The Stand Back study evaluated the} feasibility and effects of a multicomponent intervention targeting reduced prolonged sitting and pain selfmanagement in desk workers with chronic low back pain (LBP).

Methods This randomised controlled trial recruited 27 individuals with chronic LBP, Oswestry Disability Index (ODI) $>10 \%$ and desk jobs (sitting $\geq 20$ hours/ week). Participants were randomised within strata of ODI $(>10 \%-<20 \%, \geq 20 \%)$ to receive bimonthly behavioural counselling (in-person and telephone), a sitstand desk attachment, a wrist-worn activity-prompting device and cognitive behavioural therapy for LBP self-management or control. Self-reported work sitting time, visual analogue scales (VAS) for LBP and the ODI were assessed by monthly, online questionnaires and compared across intervention groups using linear mixed models.

Results Baseline mean (SD) age was 52 (11) years, $78 \%$ were women, and ODI was 24.1 (10.5)\%. Across the 6-month follow-up in models adjusted for baseline value, work sitting time was 1.5 hour/day $(P<0.001)$ lower comparing intervention to controls. Also across follow-up, ODI was on average 8 points lower in intervention versus control ( $P=0.001)$. At 6 months, the relative decrease in ODI from baseline was $50 \%$ in intervention and $14 \%$ in control $(P=0.042)$. LBP from VAS was not significantly reduced in intervention versus control, though small-to-moderate effect sizes favouring the intervention were observed (Cohen's $d$ ranged from 0.22 to 0.42 ).

Conclusion An intervention coupling behavioural counselling targeting reduced sedentary behaviour and pain self-management is a translatable treatment strategy that shows promise for treating chronic LBP in desk-bound employees.

Trial registration number NCT0224687; Pre-results.

\section{INTRODUCTION}

SLinked

- http://dx.doi.org/10.1136/ oemed-2017-104981

Check for updates

To cite: Barone Gibbs $B$, Hergenroeder AL, Perdomo SJ, et al. Occup Environ Med 2018;75:321-327.
Low back pain (LBP) is highly prevalent, with a global point prevalence estimate of $9.4 \%$, and is the leading global cause of disablement. ${ }^{1-3} \mathrm{LBP} /$ neck pain is the third-leading cause of medical expenditures in the USA and medical expenditures related to $\mathrm{LBP} /$ neck pain increased by $>100 \%$ between 1996 and 2013. ${ }^{4}$ Despite increased costs and utilisation of interventions to treat LBP, the prevalence of LBP has risen. ${ }^{5}$ Chronic LBP lasting beyond 12 weeks is especially difficult to manage and many individuals experience persistent pain despite

\section{Key messages}

What is already known about this subject?

- Preliminary studies suggest that interrupting or reducing prolonged sitting might decrease low back pain (LBP) in desk workers, though most interventions have been acute or short term and are limited to a single component.

\section{What are the new findings?}

- A 6-month multicomponent intervention targeting reduced sitting and pain selfmanagement non-significantly reduced ratings of LBP and significantly decreased LBP disability by $50 \%$ vs a $14 \%$ decrease in controls.

\section{How might it impact on clinical practice in the} foreseeable future?

- A multicomponent intervention that couples decreased workplace sitting (via behavioural intervention, a height adjustable workstation and an activity prompter) with pain selfmanagement is a simple, inexpensive treatment strategy that shows promise for reducing disability in desk workers with chronic LBP.

treatment. ${ }^{67}$ Increasing prevalence and costs associated with chronic LBP and its deleterious effects require a simple, inexpensive, evidence-based approach to better manage persistent LBP.

Non-surgical, non-pharmacological treatment approaches are recommended for treatment of chronic LBP and include exercise and cognitive behavioural therapy. ${ }^{8}$ For example, a recent meta-analysis concluded that cognitive behavioural therapy interventions (that were often pragmatic and low cost) resulted in long-term benefits for chronic LBP. ${ }^{9}$ However, the effectiveness of cognitive behavioural therapy interventions was modest, with a pooled effect size of $d=0.2,{ }^{9}$ suggesting a need for supplemental approaches. Exercise therapy is also effective for managing LBP, but also has small effects and adherence can be poor. ${ }^{10}$ Targeting a reduction in prolonged sitting or 'sedentary behaviour' may be an additional, non-invasive treatment strategy. Sedentary behaviour is distinct from a lack of exercise and is defined as 'any waking behaviour characterised by an energy expenditure $\leq 1.5$ metabolic equivalents, while in a sitting, reclining or lying posture'. ${ }^{11}$ Excessive sitting might contribute to chronic LBP 
and disability through tightening of lower extremity musculature, decreased circulation, deconditioning and poor posture, resulting in increased pain and stiffness. ${ }^{12}{ }^{13}$ Furthermore, those with chronic pain may have the tendency to avoid movement for fear of pain or reinjury ${ }^{14}$ and spend even more time being sedentary.

Desk workers are a potential target for sedentary behaviour interventions because they are prone to greater amounts of prolonged sitting ${ }^{15}$ and commonly suffer from LBP, with a 1 -year prevalence of $30 \%-50 \% .{ }^{16}{ }^{17}$ Recent studies have found that workplace interventions to decrease prolonged sedentary behaviour have decreased LBP in general, occupational samples ${ }^{18-23}$ and chronic LBP $^{24}$ Further, productivity was not reduced in these interventions, suggesting that these strategies could be acceptable for implementation in the work environment. ${ }^{18-20}$ However, these studies were mostly short term $(<12$ weeks) and did not include multicomponent interventions specifically designed to facilitate standing, movement and pain self-management in workers with desk jobs and chronic LBP.

To address these gaps, the 6-month Stand Back trial aimed to evaluate the feasibility and effect of a multicomponent intervention targeting decreased sedentary behaviour through use of a sit-stand desk attachment, movement breaks and pain self-management in desk workers with chronic LBP. The primary outcomes were LBP and disability. In addition, this study sought to evaluate feasibility and the effect of the intervention on sitting time, physical activity and physical function.

\section{METHODS}

\section{Participants and setting}

The Stand Back randomised trial recruited participants from the greater Pittsburgh area using fliers, University-based electronic mailings and University of Pittsburgh research registries from October 2015 through May of 2016. Inclusion criteria were (1) chronic LBP defined as persistent LBP for at least 3 months and resulting in pain on more than half of the days in the past 6 months $^{21}$; (2) LBP disability as defined by an Oswestry Disability Index (ODI) $>10 \%$; (3) currently performing deskwork $\geq 20$ hours/week at a desk compatible with the sit-stand desk attachment; (4) stable employment (at least 3 months at current job; plan to stay at current job for at least 6 months); (5) approval from supervisor to participate and install desk attachment and (6) access to internet to complete monthly online questionnaires. Participants were excluded if they did not provide informed consent, had a cardiovascular event in the past 6 months, had a comorbidity that limited the ability to reduce sedentary behaviour (eg, currently undergoing treatment for cancer), had recent ( $<3$ months) or planned back surgery, reported symptoms consistent with a more serious spinal condition (eg, compression fracture and infection), were currently using a height-adjustable/standing workstation or activity prompter, were currently or planning to get pregnant, or had blood pressure $\geq 160 / 100 \mathrm{~mm} \mathrm{Hg}$. Participants were screened for eligibility first by phone and then in-person. All research procedures stopped when a participant became ineligible and thus the first failed eligibility criteria are reported herein. All participants provided written informed consent to the Principal Investigator during an orientation where the study rationale and hypotheses, all research procedures, and potential risks and benefits were explained. All research procedures were approved by the University of Pittsburgh Institutional Review Board. The study was registered with clinicaltrials.gov (NCT02624687).

\section{Randomisation}

Participants were randomised 1:1 within strata of LBP disability severity (ODI $>10 \%$ to $<20 \%$ or $\geq 20 \%$ ) by non-blinded study staff. In each stratum, randomisation was assigned by the sealed envelope method in blocks of 4 by a study investigator.

\section{Intervention}

Components of the 6-month intervention included behavioural counselling, use of a sit-stand desk attachment and activity prompter to reduce prolonged sedentary behaviour, and pain self-management. Participants randomised to the intervention also received an initial in-person counselling session followed by monthly contact, via phone call, with a physical therapist or a trained interventionist.

The initial face-to-face, in-person lesson lasted 75-90 min and included education about the health risks of sedentary behaviour, behavioural counselling for reducing sitting, installation of a QuickStand sit-stand desk attachment (Humanscale, New York, New York, USA), provision of the wrist-worn activity prompting device (UP wristband, Jawbone, San Francisco, California, USA) and cognitive behavioural therapy for pain management. Though goals could be individually tailored, participants were encouraged to stand for at least 2 (and up to 4) hours/day and to alter working posture frequently (eg, every $30 \mathrm{~min}$ ) with the sit-stand desk attachment. ${ }^{25}$ Research staff programmed the wrist-worn devices with generic accounts and instructed participants only to use the activity prompting feature. Research staff set the 'idle alert' of the wrist-worn activity prompter to vibrate after $30 \mathrm{~min}$ without movement, though individual modifications were possible. Participants were encouraged to take an activity break in response to an 'idle alert' such as a 2-3 min walk, moving about their work space. Lastly, a cognitive behavioural approach was used to facilitate self-management of chronic LBP $^{26}$ The cognitive behavioural approach targeted the participant's beliefs about pain and addressed symptom management in the context of the prescribed intervention that targeted reduced sitting and increased standing and moving. Individualised goals and strategies were formulated and participants were provided with written materials and a behavioural contract.

This initial session was followed by six individual monthly phone sessions, with a target duration of 10-15 min per session, which targeted goal setting, barriers to sitting less and introduced additional aspects of behaviour change theory (ie, social support and stimulus control). ${ }^{27-29}$ Intervention participants completed a survey at the end of the study reporting satisfaction, favourable and unfavourable effects of the intervention, and ratings of helpfulness for individual intervention components.

Control participants received no intervention during the 6-month follow-up, but were offered a $60 \mathrm{~min}$ in-person lesson at the end of the study, if desired, which summarised the intervention.

All participants kept (intervention group) or received the wrist-worn activity prompter at the end of the study and up to $\$ 100$ for completing all assessments. Sit-stand desk attachments were recovered from intervention participants after the 6-month intervention and reused.

\section{Measurements}

Baseline descriptive measurements included weight by a digital scale and height by a wall-mounted stadiometer in light street clothing and with shoes removed. Demographic characteristics and use of pain medications were self-reported. 


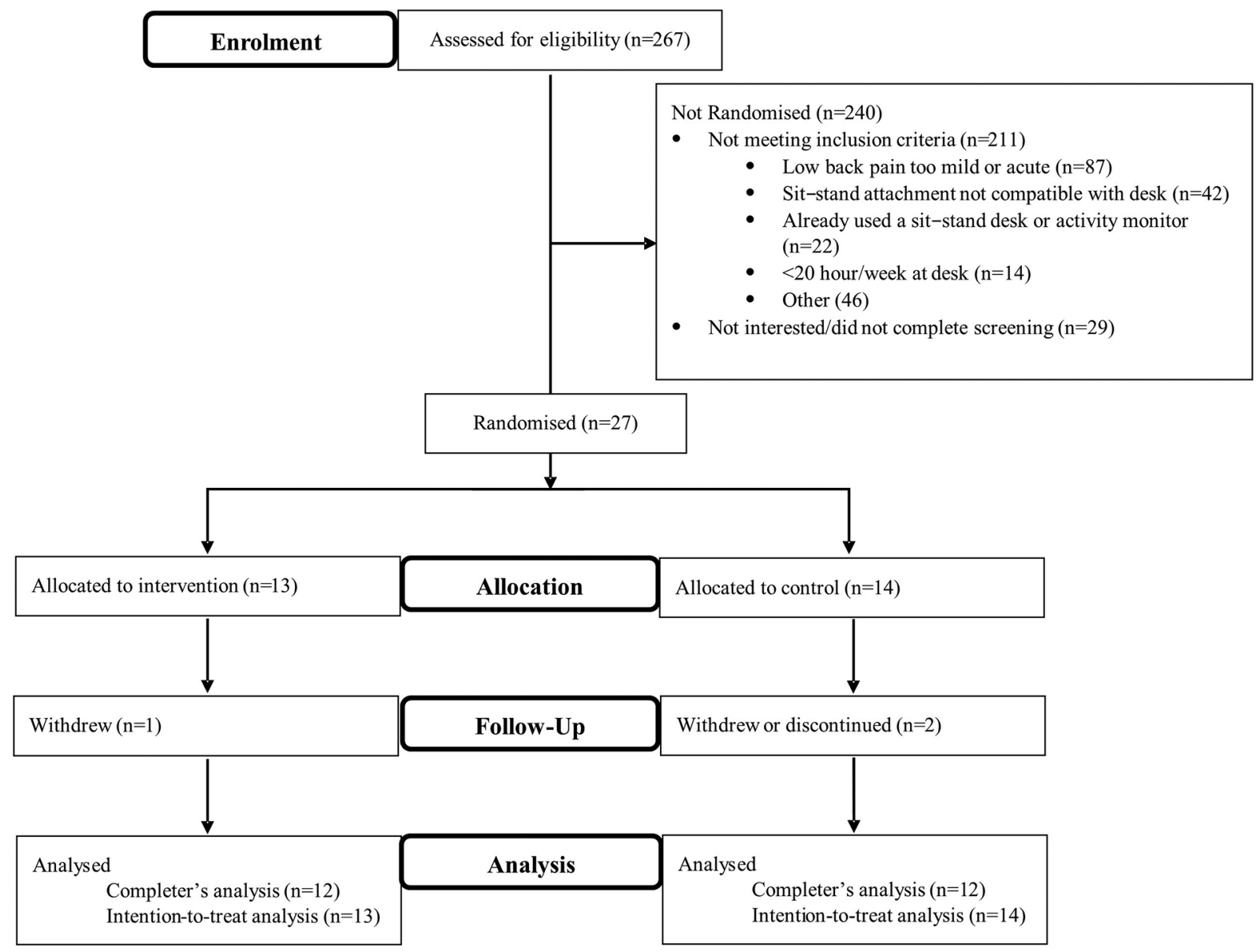

Figure 1 Participant flow diagram.

Self-reported outcomes were measured via online questionnaires (Qualtrics, Provo, Utah, USA). Pain and disability levels were assessed monthly as \% disability from the ODI (primary outcome $^{30}$ and 10-point visual analogue scales (VAS) querying current, usual, worst and best LBP in the past week. ${ }^{31}$ Usual neck, upper back and leg pain were also assessed monthly using the 10-point VAS. Participation in moderate-to-vigorous physical activity (recreational, occupational and transportation) was assessed by the Global Physical Activity Questionnaire (GPAQ) $)^{32}$ at baseline, 3 and 6 months. Those reporting a total of $\geq 150 \mathrm{~min} /$ week of moderate-to-vigorous physical activity or $\geq 75 \mathrm{~min} /$ week of vigorous physical activity were classified as meeting physical activity recommendations. ${ }^{33}$ Sedentary behaviour was assessed monthly as total hours/day spent sitting, separately at work and overall, using a question from the GPAQ. On a monthly basis, participants were also asked to report any changes in their medical history including any of the following adverse events related to their back condition: (1) $>50 \%$ increase in LBP since the beginning of the study; (2) new weakness, tingling or numbness in lower legs; and (3) any hospitalisation or injury that required medical treatment.

Physical function was measured at baseline and 6 months by blinded research personnel using a battery of standard tests found to be clinically useful in LBP including: (1) 50 -foot walk test, (2) repeated sit-to-stand, (3) timed-up-and-go and (4) unloaded/loaded reach test. ${ }^{34}$ To decrease participant burden, this assessment occurred at the participant's workplace; the chair and location used for the assessment at baseline were noted and repeated at the 6-month visit. Functional tests were performed using standardised protocols. ${ }^{35-37}$

Feasibility outcomes included recruitment and retention rates, adherence to intervention contacts and sedentary behaviour goals, and intervention acceptability ratings.

\section{Statistical analysis}

Assuming a clinically meaningful 6-point difference between groups in the primary outcome (ODI), ${ }^{38}$ and a SD of 5 points, ${ }^{39}$ a two-sided $\alpha=0.05$ and $80 \%$ power, we required $n=24$ participants ( $n=12$ per group). Though we aimed to recruit 30 participants, allowing for a dropout rate of $20 \%$, slightly fewer $(n=27)$ were randomised during the enrolment period.

Baseline characteristics were summarised by intervention group as means (SD) or frequencies and compared by independent $t$ tests or $\chi^{2}$ tests. The primary analyses retained all randomised subjects; a last observation carried forward approach for missing data resulted in replacing one observation at months 1 and 2, two observations at months 3 and 4 and three observations at months 5 and 6. Linear-mixed models with random effects were used to estimate the effects of group and time (independent variables) on outcomes (eg, ODI) with adjustment for baseline value and imbalances across randomisation groups. Physical function outcome measures with a single assessment during follow-up were compared across groups using analysis of covariance linear regression models, with adjustment for baseline value and imbalances in baseline characteristics. The magnitude of group 


\begin{tabular}{|c|c|c|}
\hline & Intervention $(n=13)$ & Control $(n=14)$ \\
\hline Age (years) & $52(9)$ & $51(13)$ \\
\hline \multicolumn{3}{|l|}{ Gender } \\
\hline Men & $2(15 \%)$ & $4(29 \%)$ \\
\hline Women & $11(85 \%)$ & $10(71 \%)$ \\
\hline \multicolumn{3}{|l|}{ Race } \\
\hline White & $10(77 \%)$ & $12(86 \%)$ \\
\hline Black & $2(15 \%)$ & $1(7 \%)$ \\
\hline Asian & $1(8 \%)$ & $1(7 \%)$ \\
\hline \multicolumn{3}{|l|}{ Education* } \\
\hline Some college or associates degree & $3(23 \%)$ & $2(14 \%)$ \\
\hline Bachelor's degree & $6(46 \%)$ & $1(7 \%)$ \\
\hline Masters or doctoral degree & $4(23 \%)$ & $11(78 \%)$ \\
\hline Body mass index $\left(\mathrm{kg} / \mathrm{m}^{2}\right)$ & $31.0(7.5)$ & $29.0(5.2)$ \\
\hline Sitting time overall (hour/day) & $9.7(2.1)$ & $10.5(1.8)$ \\
\hline Sitting time at work (hour/day) & $6.6(1.2)$ & $7.3(1.0)$ \\
\hline \multicolumn{3}{|c|}{ Moderate-to-vigorous physical activity (min/week) } \\
\hline Total & $190(163)$ & $191(175)$ \\
\hline Recreational & $107(134)$ & $126(139)$ \\
\hline Occupational & $37(84)$ & $0(0)$ \\
\hline Transportation & $46(73)$ & $66(114)$ \\
\hline $\begin{array}{l}\text { Meeting physical activity } \\
\text { recommendationst }\end{array}$ & $8(62 \%)$ & $8(57 \%)$ \\
\hline $\mathrm{ODI}(\%)$ & $23.6(10.9)$ & $24.6(10.3)$ \\
\hline Using pain medications & $2(15 \%)$ & $1(7 \%)$ \\
\hline
\end{tabular}

Data are reported as mean (SD) or $n(\%)$.

*Between group difference with $\mathrm{P}<0.05$.

tBased on total moderate-to-vigorous physical activity recommendations. ODI, Oswestry Disability Index.

differences was calculated as Cohen's $d$ by dividing the adjusted group effect by the baseline SD in the control group. Sensitivity analyses conducted using only observed data (completers only analysis) were similar to the reported results (data not shown).

\section{RESULTS}

\section{Sample recruitment and retention}

Of 267 participants assessed for eligibility, 27 were randomised (figure 1). The most common reasons for ineligibility were that the LBP was too mild or acute $(\mathrm{n}=87)$ or that the sit-stand desk attachment was not compatible with the workstation $(n=42)$. Of note, only two potential subjects were unable to provide supervisor consent during screening, suggesting high acceptability of our intervention approach in the workplace. Most participants worked in a university setting $(\mathrm{n}=21)$, with the remaining six having office locations in the greater Pittsburgh area including a home office $(n=1)$, a hospital $(n=1)$, and corporate offices $(n=4)$. Most participants worked in separate locations, with the exception of some university employees working on the same floor of a building (one cluster of 2; one cluster of 3); the investigators asked these participants not to discuss the study among each other to limit contamination. One intervention participant withdrew after 1 month for personal reasons. One control participant withdrew at 2 months for personal reasons and one control participant withdrew due to extended work leave for a medical procedure unrelated to LBP. Completion rate of monthly assessment questionnaires was $93 \%$.

Characteristics were mostly balanced across randomised groups with the exception of education, which was higher in the control group $(\mathrm{P}=0.03)$ (table 1$)$. All group comparisons were subsequently adjusted for education. Participants were, on average, 52 (SD: 9) years old, had moderate LBP disability by the ODI and were mostly white women. Participants reported about 7 hours/day of sitting while at work and 10 hours/day overall. Also, 59\% of participants reported engaging in moderate-to-vigorous intensity physical activity at recommended levels.

\section{Intervention adherence and acceptability}

Excluding the participant that withdrew, adherence to in-person and monthly telephone intervention contacts was $100 \%$, with each of these contacts lasting an average of $14 \mathrm{~min}$ (SD: $4 \mathrm{~min}$ ). Based on data collected during intervention calls, participants typically started with a goal of 2 hours/day of standing but reported standing for 2.9 (SD: 0.6) hours/day by the end of follow-up. By 6 months, participants reported taking an average of 5.5 (SD: 1.6) movement breaks per day.

In the postintervention survey, participants were extremely satisfied $(n=11,92 \%)$ or satisfied $(n=1,8 \%)$ with the overall intervention. Additionally, intervention participants reported benefits of the intervention with the following frequencies: less pain (92\%), greater productivity (67\%), healthier (58\%), more energy $(50 \%)$, more focus $(50 \%)$, greater comfort $(50 \%)$, happier (42\%) and less stress (33\%). No participant reported unfavourable effects of the intervention on their health or mood. Participants also rated the helpfulness of individual components of the intervention on a 5 -point scale with 5 being the best as follows: sit-stand desk attachment=4.8 (SD: 0.4), activity prompter $=3.8(\mathrm{SD}: 1.5)$, pain management strategies $=4.1(\mathrm{SD}:$ 0.9 ) and follow-up phone calls=4.3 (SD: 0.9).

\section{Sedentary time and physical activity}

Self-reported sitting time was significantly lower in the intervention versus control group during follow-up (figure 2, numbers reported in the online supplementary appendix table 1). Averaged across follow-up, intervention participants sat less than controls $(-1.4$ hour/day, $\mathrm{P}=0.02, d=0.78)$ after adjustment for baseline value and education. Specifically during work hours, intervention participants sat 1.5 hour/day less than controls $(\mathrm{P}<0.001, d=1.36)$. At 6 months, intervention versus control participants had decreased their all-day sitting time by $30 \%$ versus $15 \%(\mathrm{P}=0.008)$ and their work sitting by $35 \%$ versus $14 \%(\mathrm{P}=0.014)$. Total moderate-to-vigorous intensity physical activity was non-significantly higher with a small-to-moderate effect size in intervention versus control subjects over follow-up $(\beta=56 \mathrm{~min} /$ week, $\mathrm{P}=0.474, d=0.32)$; specific subcategories were also not statistically difference between groups categories (online supplementary table 1 ).

\section{LBP disability}

LBP disability as measured by the ODI significantly decreased in the intervention versus control group (figure 3, numbers reported in the online supplementary appendix table 1), averaging an adjusted 8-point difference across all follow-up visits $(\mathrm{P}=0.001, d=0.73)$. At 6 months, the relative decrease from baseline in ODI was 50\% among intervention participants and $14 \%$ among controls $(\mathrm{P}=0.042)$. Though usual self-reported LBP, neck pain, upper back pain and leg pain on a 10-point VAS were lower in the intervention group versus the control group across follow-up ( $d$ were small-to-moderate and ranged from 0.22 to 0.52 ), the overall group differences were not statistically significant in adjusted models $(\mathrm{P}>0.05$, online supplementary appendix table 2). 

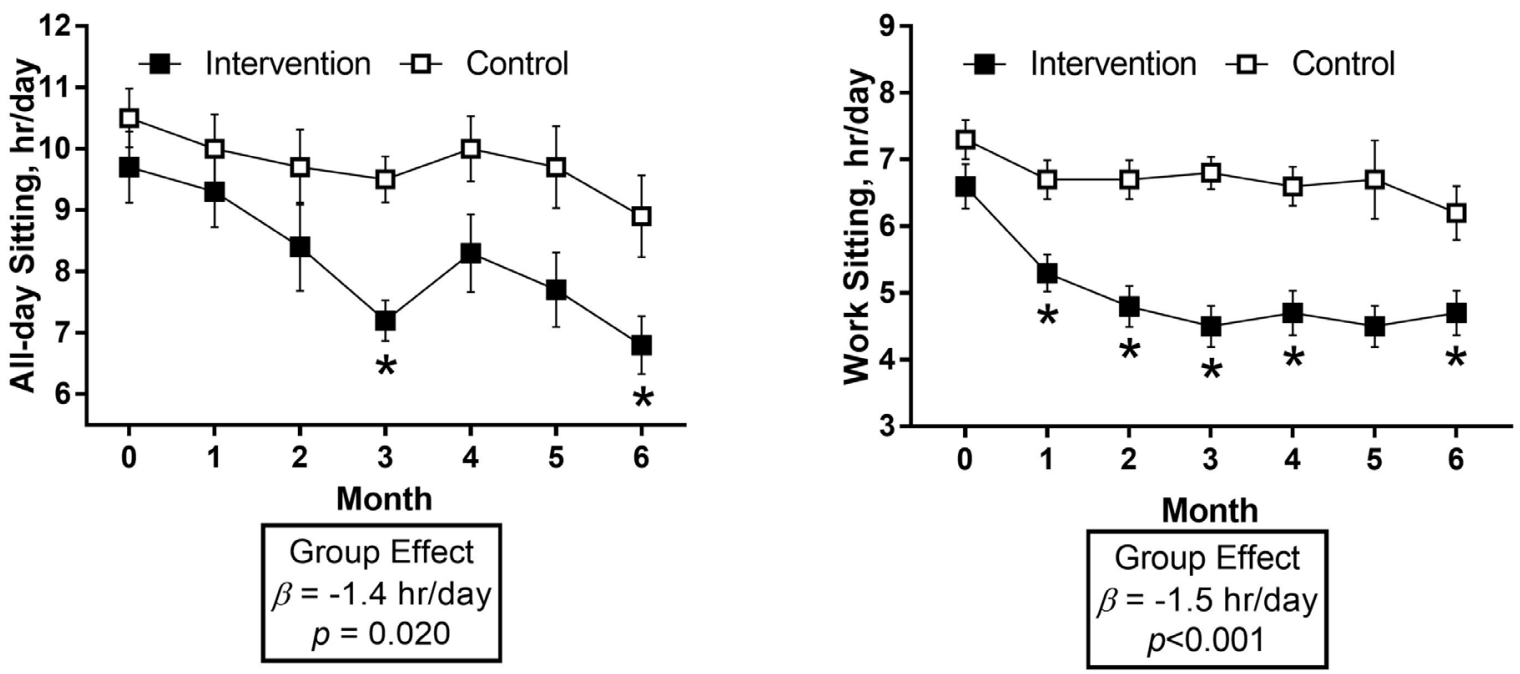

Figure 2 Sitting time by intervention group. Plotted values are mean \pm SEM. Numerical data reported in the online supplementary appendix table 1. Group effect calculated from a linear mixed model adjusting for baseline value, time and education. ${ }^{*}$ Difference between groups with $\mathrm{P}<0.05$ at specific month.

\section{Physical function}

Physical function tests were performed at the beginning and end of the 6-month study period (table 2). No statistically significant group differences were observed. However, a potential benefit of the intervention was suggested with small-to-moderate effect sizes for sit-to-stands $(d=0.24, \mathrm{P}=0.226)$ and the unloaded reach $(d=0.48, \mathrm{P}=0.245)$.

\section{Adverse events}

When asked whether LBP had increased $>50 \%$ since the beginning of the study at monthly assessments, three (21\%) control participants and one (8\%) intervention participant reported such an increase; the intervention participant did not attribute the increase in pain to the intervention (due to carrying heavy luggage). One (7\%) control participant reported new weakness, numbness or tingling in the lower legs. One (7\%) control

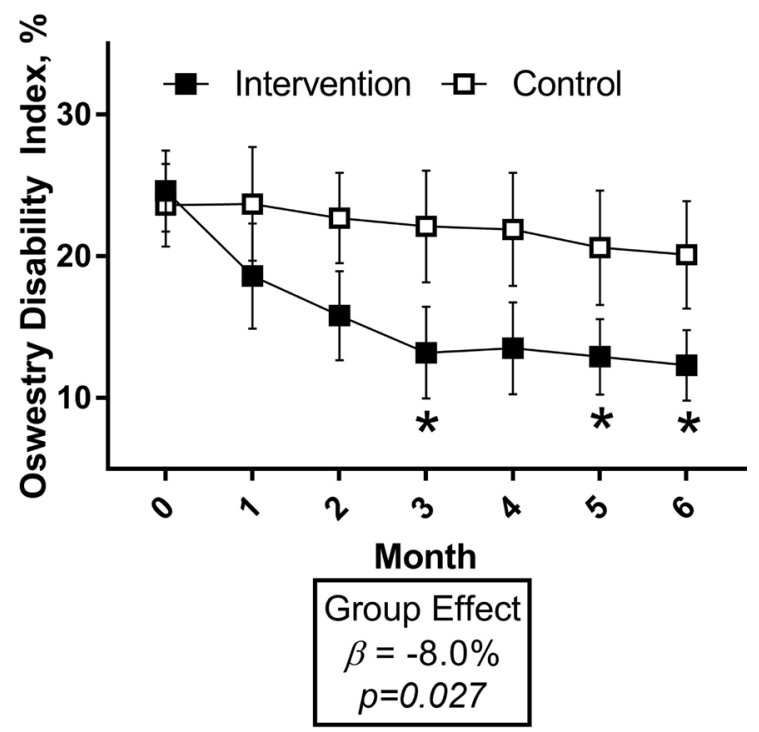

Figure 3 ODI by the intervention group. Plotted values are mean \pm SEM. Numerical data reported in the online supplementary appendix table 1. Group effect calculated from a linear mixed model adjusting for baseline value, time and education. * Difference between groups with $\mathrm{P}<0.05$ at specific month. ODI, Oswestry Disability Index. participant was hospitalised for an unrelated procedure. No injuries related to the intervention were reported.

\section{DISCUSSION}

The Stand Back intervention resulted in high adherence and ratings of satisfaction/perceived benefits, reduced sedentary time and shows promise for improving LBP and related disability in working adults with desk jobs and chronic LBP. LBP disability was significantly reduced in intervention versus control participants. The study was not powered sufficiently to expect statistically significant decreases in self-rated pain and physical function, however small-to-moderate beneficial effects suggest that the intervention could improve these outcomes if studied on a larger scale. These improvements may have contributed to the observed reduction in LBP disability. The results of this study inform the feasibility of our intervention and support that targeting sedentary behaviour in this population is a potentially effective treatment strategy for chronic LBP.

Table 2 Baseline and 6-month physical function by the intervention group

\begin{tabular}{|c|c|c|c|c|c|}
\hline & Group & Baseline & 6 months & $\begin{array}{l}\text { Group } \\
\text { effect }\end{array}$ & $d$ \\
\hline Sit-to-stands (s) & $\begin{array}{l}\text { Intervention } \\
\text { Control }\end{array}$ & $\begin{array}{l}11.3(2.0) \\
11.2(2.6)\end{array}$ & $\begin{array}{l}10.3(2.2) \\
10.6(2.7)\end{array}$ & $\begin{array}{l}\beta=-0.63 \\
P=0.226\end{array}$ & 0.24 \\
\hline $\begin{array}{l}\text { Fifty foot walk - } \\
\text { usual pace (s) }\end{array}$ & $\begin{array}{l}\text { Intervention } \\
\text { Control }\end{array}$ & $\begin{array}{l}13.6(1.7) \\
14.1(2.5)\end{array}$ & $\begin{array}{l}13.4(1.7) \\
14.0(2.9)\end{array}$ & $\begin{array}{l}\beta=0.06 \\
P=0.913\end{array}$ & 0.02 \\
\hline $\begin{array}{l}\text { Fifty foot walk - } \\
\text { fast pace (s) }\end{array}$ & $\begin{array}{l}\text { Intervention } \\
\text { Control }\end{array}$ & $\begin{array}{l}10.5(1.2) \\
11.1(2.0)\end{array}$ & $\begin{array}{l}10.7(1.3) \\
11.1(2.2)\end{array}$ & $\begin{array}{l}\beta=0.07 \\
P=0.898\end{array}$ & 0.04 \\
\hline $\begin{array}{l}\text { Timed up-and- } \\
\text { go (s) }\end{array}$ & $\begin{array}{l}\text { Intervention } \\
\text { Control }\end{array}$ & $\begin{array}{l}7.8(0.7) \\
8.3(1.6)\end{array}$ & $\begin{array}{l}7.6(1.1) \\
8.1(1.4)\end{array}$ & $\begin{array}{l}\beta=0.25 \\
P=0.421\end{array}$ & 0.15 \\
\hline $\begin{array}{l}\text { Unloaded reach } \\
(\mathrm{cm})\end{array}$ & $\begin{array}{l}\text { Intervention } \\
\text { Control }\end{array}$ & $\begin{array}{l}33.6(6.8) \\
33.9(5.1)\end{array}$ & $\begin{array}{l}36.1(7.3) \\
34.5(4.8)\end{array}$ & $\begin{array}{l}\beta=2.45 \\
P=0.245\end{array}$ & 0.48 \\
\hline Loaded reach $(\mathrm{cm})^{*}$ & $\begin{array}{l}\text { Intervention } \\
\text { Control }\end{array}$ & $\begin{array}{l}23.5(6.7) \\
23.4(7.8)\end{array}$ & $\begin{array}{l}24.0(7.1) \\
23.4(5.7)\end{array}$ & $\begin{array}{l}\beta=0.43 \\
P=0.856\end{array}$ & 0.06 \\
\hline
\end{tabular}

Data displayed as mean (SD).

$d$ is Cohen's effect size.

$\beta$ coefficients and $\mathrm{p}$-values from linear regression models including group, time, education and baseline value as covariates.

${ }^{*}$ One control participant was unable to lift weight during loaded reach test at either assessment. 
This robust effect on LBP disability observed in our study that was apparent by 3 months suggests that our multicomponent intervention targeting sedentary behaviour appears to be more effective compared with studies using individual components treating LBP. A meta-analysis pooled the long-term ( $>6$ months) effects of cognitive-behavioural therapy versus usual care for LBP and found small reductions in pain $(\mathrm{n}=10$ studies, $d=0.22$, $\mathrm{P}<0.05)$ and disability $(\mathrm{n}=7$ studies, $\mathrm{d}=0.19, \mathrm{P}<0.05){ }^{9}$ These are notably smaller than the effects of the Stand Back multicomponent intervention on LBP disability $(d=0.73)$ and comparable or smaller than the effects of the Stand Back intervention on pain ( $d$ ranging from 0.22 to 0.53 ). Our findings can also be compared with a 12 week trial by Oglibene et al, that randomised 57 desk workers with chronic LBP to either installation of a sit-stand desk attachment (without further intervention) or control. ${ }^{24}$ Among the 46 participants completing the study, those receiving sit-stand desk attachments had significantly reduced LBP as compared with controls based on daily logs using the VAS. Though this study used a different instrument (Roland Morris Disability Questionnaire) and was shorter in length (3 vs 6 months), Oglibene et al did not find a decrease in LBP disability. Though our multicomponent intervention appears to have yielded greater benefits for LBP disability, a direct comparison of individual versus multicomponent intervention strategies in this population is essential for future research.

A growing number studies suggest that breaking up prolonged sitting with standing or movement breaks can provide immediate benefits to pain and discomfort and provide insight into the long-term results we observed in the Stand Back pilot trial. Kowalsky et $a l^{40}$ and Thorp et $a l^{18}$ have found that alternating between standing and sitting postures while performing deskwork in a laboratory setting acutely reduces reported LBP in working age adults. Sedentary behaviour interventions in working populations in office ${ }^{18-204142}$ or community ${ }^{43}$ settings have also decreased self-reported pain. Of importance in the context of adding an option to perform desk work in a standing position, prolonged, static standing is also related to adverse health outcomes, such as incidence of varicose veins and LBP. $^{22} 2344$ Combining the evidence that both prolonged sitting and prolonged standing appear to increase pain and discomfort, these studies are consistent with our findings that increasing postural changes and movement breaks can reduce LBP and disability.

Moreover, most participants were physically active at recommended levels ${ }^{33}$ at baseline and the effect of the intervention on moderate-to-vigorous physical activity was not statistically significant. These data suggest that specifically targeting reduced sedentary behaviour is a unique strategy, distinct from increasing moderate-to-vigorous physical activity, that could be effective for chronic LBP management. Though our measurements are not ideal for conducting compositional data analysis, our data suggest that sitting was replaced by increased standing at the sit-stand desk attachment, movement breaks, and possibly (though non-significantly) moderate-to-vigorous intensity physical activity. Further, participants in the intervention were specifically counselled to sit less by standing and taking movement breaks at work and home (and increasing moderate-to-vigorous intensity physical activity behaviour was specifically not discussed). Future research with objectively monitored sitting and physical activity and using a compositional data analysis approach will improve our understanding of the optimal replacement behaviours to sitting that yield benefits to LBP disability.

These data also provide information relevant to the feasibility and acceptability of our multicomponent intervention.
Though most participants were university employees, office settings ranged from cubicles to offices with doors, suggesting our intervention might be applied in a variety of workplaces. All components of the intervention were rated as helpful by participants, though scores ranged from 3.8 (activity prompter) to 4.8 (sit-stand desk attachment) on a 5-point scale. Other positive feasibility aspects include the high adherence to intervention contacts and targets, acceptability and retention of participants. However, many potential subjects were excluded for having LBP below our preset threshold for duration or severity or a workstation that could not accommodate our sit-stand attachment. Future research could consider including participants with less severe LBP and using more adaptable sit-stand desk attachments to be more inclusive.

Strengths of this study include the translatable intervention with a single in-person visit followed by monthly phone calls that could be easily replicated through the use of health coaches. The randomised design is also a strength, as the observed intervention effect was beyond reductions in sitting and LBP disability reported by the control group over follow-up. These effects in the no-treatment control group may have resulted from regression to the mean (inclusion criteria were sitting at desk for $\geq 20$ hours/ week and ODI $>10 \%$ ), exposure to the study rationale during screening or general media attention to the potential health risks of sitting. Other strengths include the 6-month duration, and a study sample with a range of LBP disability, body mass index and physical activity levels. Also, our flexible intervention was rated highly across an array of work settings-some of which required that participants almost exclusively stay at their desk. Limitations of the current research include the small sample size, measurement of sitting time and physical activity by self-report, and the inability to evaluate the effects of individual components of the intervention on outcomes. Further, results could be vulnerable to bias resulting from an imbalance in contact time between the intervention and the no-treatment control groups or from expectancy due to the recent media attention on the potential harms of excessive sitting.

The Stand Back multicomponent intervention, targeting reduced sitting through height adjustable workstations and activity prompting devices combined with pain self-management, had high feasibility and shows promise for the treatment of LBP among desk workers. Chronic LBP is a prevalent, debilitating and costly condition; our novel intervention strategy is a potential additional, conservative treatment strategy. Moreover, with more flexibility regarding the type of height-adjustable workstation, our intervention is likely acceptable across a wide range of work settings and desk-based professions. Future studies that are larger use objective monitoring of sedentary behaviour and activity, and evaluate intervention components separately will be important for designing cost-effective interventions for desk workers with chronic LBP. Measuring the effect of the intervention on productivity, work satisfaction and well-being are additionally important areas for future research.

Acknowledgements The authors would like to thank the participants of the Stand Back study as well as the efforts of research assistants Marco Pugliese, MS, John Michael Taormina, BS, and Mary Duggan, BS.

Contributors BBG, ALH, AD and JMJ: conceived the idea. BBG, ALH and SJP: designed and delivered the intervention. BBG: wrote the manuscript and performed the statistical analysis. ALH, SJP, RJK, AD and JJ: reviewed the manuscript and provided critical feedback and approval for submission.

Funding This study was funded by the Virginia Kaufman Endowment Fund for pain research at the University of Pittsburgh Clinical and Translational Science Institute (CTSI) at the University of Pittsburgh. In addition, recruitment support through the University of Pittsburgh CTSI was funded by the National Institutes of Health 
(UL1TR000005). ALH reports receiving funding from the National Institutes of Health as a Postdoctoral Scholar awarded to the University of Pittsburgh Pepper Center. AD reports being site Principal Investigator and Program Director for funding from the National Institutes of Health and Principal Investigator for funding from the Patient Centered Outcomes Research Institute.

Competing interests BBG reports being a Principal Investigator on studies funded by the National Institutes of Health, and the American Heart Association, and the Tomayko Foundation, and a Co-Investigator on studies funded by the Agency for Healthcare and Quality Research and the National Institutes of Health. BBG was also the Principal Investigator of a separate, investigator-initiated study funded by the Humanscale company; Humanscale had no role and provided no funding or supplies to this research. JMJ reports being one the Scientific Advisory Board for Weight Watchers International, a Co-Investigator on a study funded by Weight Watchers International awarded to the University of Pittsburgh, a Co-Investigator on a study funded by Humanscale awarded to the University of Pittsburgh, and a Principal and Co-Investigator on studies funded by the National Institutes of Health awarded to the University of Pittsburgh. JMJ was also a Co-Investigator of a separate, investigator-initiated study funded by the Humanscale company; Humanscale had no role and provided no funding or supplies to this research.

Patient consent Obtained.

Ethics approval University of Pittsburgh Institutional Review Board.

Provenance and peer review Not commissioned; externally peer reviewed.

(c) Article author(s) (or their employer(s) unless otherwise stated in the text of the article) 2018. All rights reserved. No commercial use is permitted unless otherwise expressly granted.

\section{REFERENCES}

1 Institute of Medicine (U.S.) Committee on Advancing Pain Research Care and Education. Relieving pain in America: a blueprint for transforming prevention, care, education, and research. Washington, DC: National Academies Press, 2011.

2 Lawrence RC, Felson DT, Helmick CG, et al. Estimates of the prevalence of arthritis and other rheumatic conditions in the United States. Part II. Arthritis Rheum 2008;58:26-35.

3 Hoy D, March L, Brooks P, et al. The global burden of low back pain: estimates from the global burden of disease 2010 study. Ann Rheum Dis 2014;73:968-74.

4 Reinhardt UE, Hussey PS, Anderson GF. U.S. health care spending in an international context. Health Aff 2004;23:10-25.

5 Freburger JK, Holmes GM, Agans RP, et al. The rising prevalence of chronic low back pain. Arch Intern Med 2009;169:251-8.

6 Cottingham JT, Maitland J. A three-paradigm treatment model using soft tissue mobilization and guided movement-awareness techniques for a patient with chronic low back pain: a case study. J Orthop Sports Phys Ther 1997;26:155-67.

7 Chou R, Qaseem A, Snow V, et al. Diagnosis and treatment of low back pain: a joint clinical practice guideline from the American College of Physicians and the American Pain Society. Ann Intern Med 2007;147:478-91.

8 Qaseem A, Wilt TJ, McLean RM, et al. Noninvasive treatments for acute, subacute, and chronic low back pain: a clinical practice guideline from the american college of physiciansnoninvasive treatments for acute, subacute, and chronic low back pain. Annals of Internal Medicine 2017.

9 Richmond H, Hall AM, Copsey B, et al. The effectiveness of cognitive behavioural treatment for non-specific low back pain: a systematic review and meta-analysis. PLoS One 2015;10:e0134192.

10 van Tulder M, Malmivaara A, Esmail R, et al. Exercise therapy for low back pain: a systematic review within the framework of the cochrane collaboration back review group. Spine 2000;25:2784-96.

11 Tremblay MS, Aubert S, Barnes JD, et al. Sedentary Behavior Research Network (SBRN) - terminology consensus project process and outcome. Int J Behav Nutr Phys Act 2017; $14: 75$.

12 Dankaerts W, O'Sullivan P, Burnett A, et al. Differences in sitting postures are associated with nonspecific chronic low back pain disorders when patients are subclassified. Spine 2006;31:698-704

13 Hodges PW, Moseley GL. Pain and motor control of the lumbopelvic region: effect and possible mechanisms. J Electromyogr Kinesiol 2003;13:361-70.

14 Vlaeyen JW, Kole-Snijders AM, Boeren RG, et al. Fear of movement/(re)injury in chronic low back pain and its relation to behavioral performance. Pain 1995;62:363-72.

15 Saidj $M$, Menai $M$, Charreire $H$, et al. Descriptive study of sedentary behaviours in 35,444 French working adults: cross-sectional findings from the ACTI-Cités study. BMC Public Health 2015;15:379

16 Spyropoulos P, Papathanasiou G, Georgoudis G, et al. Prevalence of low back pain in greek public office workers. Pain Physician 2007;10:651-9.
17 Jin K, Sorock GS, Courtney TK. Prevalence of low back pain in three occupational groups in Shanghai, People's Republic of China. J Safety Res 2004;35:23-8.

18 Thorp AA, Kingwell BA, Owen N, et al. Breaking up workplace sitting time with intermittent standing bouts improves fatigue and musculoskeletal discomfort in overweight/obese office workers. Occup Environ Med 2014;71:765-71.

19 Pronk NP, Katz AS, Lowry M, et al. Reducing occupational sitting time and improving worker health: the Take-a-Stand Project, 2011. Prev Chronic Dis 2012;9:E154.

20 Davis KG, Kotowski SE. Postural variability: an effective way to reduce musculoskeletal discomfort in office work. Hum Factors 2014;56:1249-61.

21 Deyo RA, Dworkin SF, Amtmann D, et al. Report of the NIH Task Force on research standards for chronic low back pain. Pain Med 2014;15:1249-67.

22 Tüchsen $\mathrm{F}$, Hannerz $\mathrm{H}$, Burr $\mathrm{H}$, et al. Prolonged standing at work and hospitalisation due to varicose veins: a 12 year prospective study of the Danish population. Occup Environ Med 2005;62:847-50

23 Tissot F, Messing K, Stock S. Studying the relationship between low back pain and working postures among those who stand and those who sit most of the working day. Ergonomics 2009;52:1402-18.

24 Ognibene GT, Torres W, von Eyben R, et al. Impact of a sit-stand workstation on chronic low back pain: results of a randomized trial. J Occup Environ Med 2016;58:287-93.

25 Buckley JP, Hedge A, Yates T, et al. The sedentary office: a growing case for change towards better health and productivity. Expert statement commissioned by Public Health England and the Active Working Community Interest Company. British journal of sports medicine 2015:49:1357-62.

26 Otis J. Managing chronic pain: a cognitive-behavioral therapy approach. Oxford: Oxford university press, 2007.

27 House JS, Landis KR, Umberson D. Social relationships and health. Science 1988;241:540-5.

28 Bandura A. Health promotion from the perspective of social cognitive theory. Psychol Health 1998;13:623-49.

29 Prochaska JO, Velicer WF. The transtheoretical model of health behavior change. Am J Health Promot 1997; 12:38-48.

30 Fairbank JCT, Pynsent PB. The Oswestry disability index. Spine 2000;25:2940-53.

31 Hawker GA, Mian S, Kendzerska T, et al. Measures of adult pain: Visual Analog Scale for Pain (VAS Pain), Numeric Rating Scale for Pain (NRS Pain), McGill Pain Questionnaire (MPQ), Short-Form McGill Pain Questionnaire (SF-MPQ), Chronic Pain Grade Scale (CPGS), Short Form-36 Bodily Pain Scale (SF-36 BPS), and Measure of Intermittent and Constant Osteoarthritis Pain (ICOAP). Arthritis Care Res 2011;63(Suppl 11):S240-52.

32 Bull FC, Maslin TS, Armstrong T. Global physical activity questionnaire (GPAQ): nine country reliability and validity study. J Phys Act Health 2009;6:790-804.

33 Andersson GB. Epidemiological features of chronic low-back pain. Lancet 1999;354:581-5.

34 Simmonds MJ, Olson SL, Jones S, et al. Psychometric characteristics and clinical usefulness of physical performance tests in patients with low back pain. Spine 1998:23:2412-21.

35 Harding VR, Williams AC, Richardson PH, et al. The development of a battery of measures for assessing physical functioning of chronic pain patients. Pain 1994;58:367-75.

36 Podsiadlo D, Richardson S. The timed "Up \& Go": a test of basic functional mobility for frail elderly persons. J Am Geriatr Soc 1991;39:142-8.

37 Simmonds MJ, Claveau Y. Measures of pain and physical function in patients with low back pain. Physiother Theory Pract 1997;13:53-65.

38 Fairbank JC, Pynsent PB. The Oswestry Disability Index. Spine 2000;25:2940-53. discussion 2952.

39 Castro-Sánchez AM, Lara-Palomo IC, Matarán-Peñarrocha GA, et al. Kinesio taping reduces disability and pain slightly in chronic non-specific low back pain: a randomised trial. J Physiother 2012;58:89-95.

40 Kowalsky RJ, Perdomo SJ, Balzer JR, et al. The influence of a sit-stand desk on sleepiness, physical discomfort, physical fatigue and mental fatigue. Med Sci Sports Exerc 2017:49:844.

41 Foley B, Engelen L, Gale J, et al. Sedentary behavior and musculoskeletal discomfort are reduced when office workers trial an activity-based work environment. J Occup Environ Med 2016;58:924-31.

42 Gao Y, Nevala N, Cronin NJ, et al. Effects of environmental intervention on sedentary time, musculoskeletal comfort and work ability in office workers. Eur J Sport Sci 2016;16:747-54

43 Barone Gibbs B, Brach JS, Byard T, et al. Reducing sedentary behavior versus increasing moderate-to-vigorous intensity physical activity in older Adults. J Aging Health 2017;29:247-67.

44 Callaghan JP, De Carvalho D, Gallagher K, et al. Is Standing the Solution to Sedentary Office Work? Ergonomics in Design: The Quarterly of Human Factors Applications 2015;23:20-4. 


\section{Correction: Reducing sedentary behaviour to decrease chronic low back pain: the stand back randomised trial}

Barone Gibbs B, Hergenroeder AL, Perdomo SJ, et al. Reducing sedentary behaviour to decrease chronic low back pain: the stand back randomised trial. Occup Environ Med 2018;75:321-7.

There is an error in the clinical trials registration number listed in this article. The correct number is: NCT02624687.

(c) Article author(s) (or their employer(s) unless otherwise stated in the text of the article) 2018. All rights reserved. No commercial use is permitted unless otherwise expressly granted.

Occup Environ Med 2018;75:474. doi:10.1136/oemed-2017-104732corr1

D) Check for updates 